\title{
Wilckodontics Combined with A-PRF and i-PRF: An Interdisciplinary Approach
}

\author{
Daliah Ruth, Janani Karthick, Jaideep Mahendra, Vijayalakshmi Rajaram \\ Department of Periodontology, Meenakshi Ammal Dental College \& Hospital, Meenakshi Academy of Higher Education and \\ Research, Maduravoyal, Chennai, Tamil Nadu, India.
}

\section{Corresponding Author:}

Dr. Jaideep Mahendra

Email: jaideep_m_23@yahoo.co.in

This is an Open Access article distributed under the terms of the Creative Commons Attribution License (creativecommons.org/ licenses/by/3.0).

Received Accepted Published

December 10, 2019

February 17, 2020

March 5, 2020

\begin{abstract}
Background: Alveolar osteogenic orthodontics or periodontally accelerated osteogenic orthodontics (PAOO) is an emerging technology in the field of dentistry. It is relatively new treatment in the dental realm that combines selective alveolar corticotomy, particulate bone grafting and the application of orthodontic forces. Case Report: It is an interdisciplinary treatment approach utilizing tissue engineering principles with periodontal regenerative surgery to create rapid orthodontic tooth movement and reduce side effects like root resorption, relapse, inadequate basal bone and bacterial time-load factors. Conclusion: This case report aims at comparing the periodontally accelerated osteogenic orthodontic approach with advanced-platelet rich fibrin (A-PRF) on one side and injectable-platelet rich fibrin (i-PRF) on the other side by a split-mouth design.
\end{abstract}

Keywords: Bone Transplantation, Orthodontics, Osteogenesis, Root Resorption, Tooth Movement Techniques.

\section{Introduction}

Wilckodontics is an interdisciplinary approach, which is also known as periodontally accelerated osteogenic orthodontics (PAOO) or alveolar osteogenic orthodontics, in which orthodontic movement is enhanced by alveolar corticotomy to increase the rate of tooth movement. The alveolar bone is a mineralized structure that easily remodels than cementum, thereby allowing orthodontic tooth movement 2-3 times faster in $1 / 3^{\text {rd }}$ to $1 / 4^{\text {th }}$ the time required. However, this procedure basically focuses on bone healing pattern known as the regional acceleratory phenomenon (RAP) [1]. Thus it satisfies the protocol of periodontal tissue engineering and regenerative surgery and reduces side effects like root resorption, relapses, inadequate basal bone and infection. Tissue engineering and regeneration is a method to promote a material-induced tissue reaction that has an effective wound-healing process which is obtained by certain biomaterial that is generated from autologous blood. Platelet-rich plasma (PRP) [2] was the first generation platelet concentrate derived from human blood samples with common denominators such as the addition of anticoagulants and bovine serum which was achieved by double-centrifugation. However, bovine thrombin and various other anticoagulants limit the clinical application of PRP by suppressing the tissue regeneration and produce health hazards. For these reasons, platelet rich fibrin (PRF), a second generation platelet concentrate was developed as the first source of autogenous bloodderived growth factors harvested without the use of anti-coagulants by Choukran [3]. PRF serves as a scaffold for tissue regeneration by bearing the feature of acting as a barrier membrane in guided bone and tissue regeneration (GBR, GTR) procedures while simultaneously holding a number of growth factors responsible for wound 
healing [4]. To optimize the amount of growth factors in PRF, the third generation concentrates A-PRF evolved by decreasing the rotational speed (rpm), and increasing the centrifugation time [5] which allows the amplification of neutrophilic granulocytes, also increase the transformation of monocytes into macrophages, hence amplifying the bone stimulation effect [6]. The use of platelet concentrate in liquid (injectable) or polymerized (clot) forms can be either utilized alone or combined easily with various biomaterials that facilitate increased fibroblast production and aims at rapid wound healing [7]. In the present case, the third generation platelet concentrates A-PRF and i-PRF were used and the accelerated tooth movement was compared at the osteotomy site reinforced with A-PRF on one side and i-PRF on other side.

\section{Case Report}

A 20-years old male patient reported to the Department of Orthodontics with the chief complaint of forwardly placed teeth. Initial orthodontic intra-oral examination revealed Angle's Class II malocclusion. The patient was systemically healthy with no deleterious habits. Presurgical orthodontic treatment was initiated. The first maxillary premolars were extracted to create a space for the alignment of the proclined anterior teeth. Strap-up was carried out using pre-adjusted edgewise appliance system (MBT 0.022"). Initial alignment and leveling was started with preformed 0.016" NiTiarchwires (3M unitek) followed by $0.016 " \times 0.022 "$ NiTiarchwires and sequentially $0.017 " \times 0.025$ " NiTiarchwires (3M Unitek) and finally shifted to $0.017 " \times 0.025$ " stainless steel. This phase of treatment was continued till all the teeth were aligned. The time taken for the presurgical orthodontic phase was 3 months. The post-operative review after 3 months revealed the complete closure of the anterior region, however retraction space was still persisting. Hence, the patient was referred to the Department of periodontics for retraction space closure by corticotomy and hence became an indication for PAOO procedure [Fig. 1]. The treatment was planned to undergo PAOO combined with A-PRF on one side and PAOO with i-PRF on other side to provoke faster tooth movement and rapid wound healing by growth factors. Treatment protocol was explained and informed consent was obtained from the patient.

Before the surgery routine protocol of oral prophylaxis was done and oral hygiene instructions were given. For A-PRF preparation $10 \mathrm{ml}$ of blood was centrifuged at $1500 \mathrm{rpm}$ for 14 mins. After careful removal of the red blood cell fraction with scissors, the A-PRF clot was procured. For i-PRF preparation two tubes of $10 \mathrm{ml}$ of whole blood without anticoagulant was centrifuged at $700 \mathrm{rpm}$ for 3 mins at room temperature by Duo Centrifuge (Process for PRF, Nice, France). The upper liquid layer was collected as i-PRF.

Perioral and intraoral region was disinfected with betadine solution. After administering local anaesthesia [Fig.2] a full thickness mucoperiosteal flap was elevated in relation to 23 to 25 region [Fig.3], which was extended $2-3 \mathrm{~mm}$ beyond the mucogingival junction. Vertical grooves were placed in the interradicular space between the root prominences with the help of bone cutting burs and adequate saline irrigation. The vertical cuts extended $2-3 \mathrm{~mm}$ from the alveolar crest to approximately $2 \mathrm{~mm}$ beyond the apices of the roots. Perforations were made in the alveolar bone over the radicular surfaces with round bur

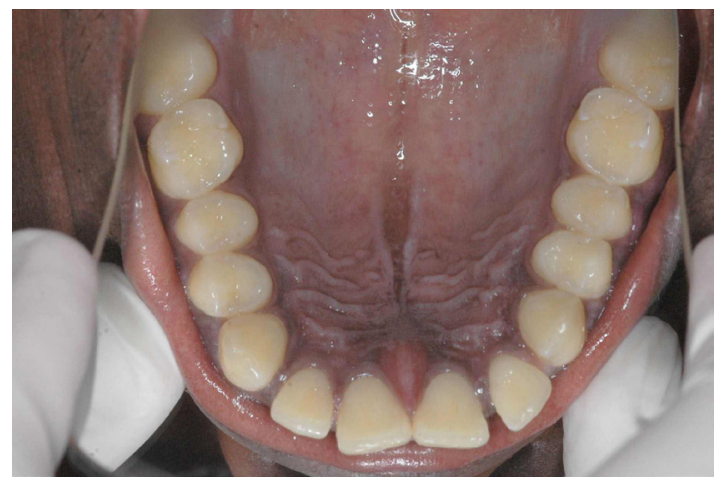

Fig.1: Pre-operative view before extraction. 
[Fig.4]. The procured A-PRF gel was mixed with osseograft (xenograft) [Fig.5] and placed in the surgical site [Fig.6]. The flap was adapted without tension to the original position and sutured with 4-0 silk sutures [Fig.7]. The sutures were left in place for ten days [Fig.8].

After one week, a similar technique was done in relation to 13 to 15 region. In this region, the procured i-PRF was mixed with osseograft (xenograft) [Fig.9] and placed at the surgical site and the flap sutured. Post-operative instructions were given during each session of surgery followed by prescription of antibiotics and analgesics. Sutures were removed after ten days. Orthodontic treatment was started 2 weeks after surgery, 250 grams of force was applied on both sides and appliance was activated every 2 weeks. At the end of three months the space was completely closed [Fig.10].

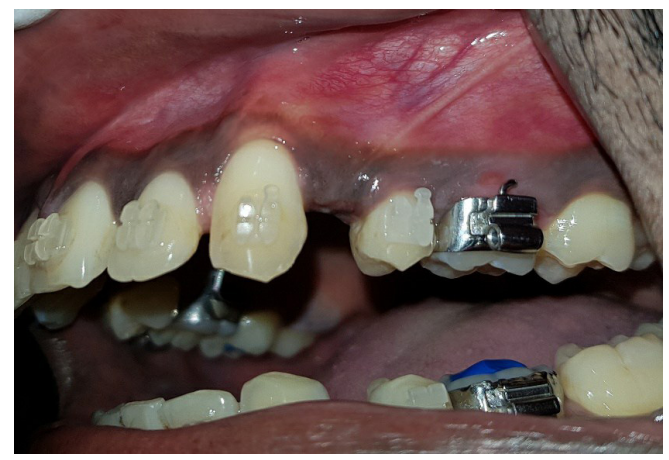

Fig.2: Pre-operative view i.r.t 23-25 region.

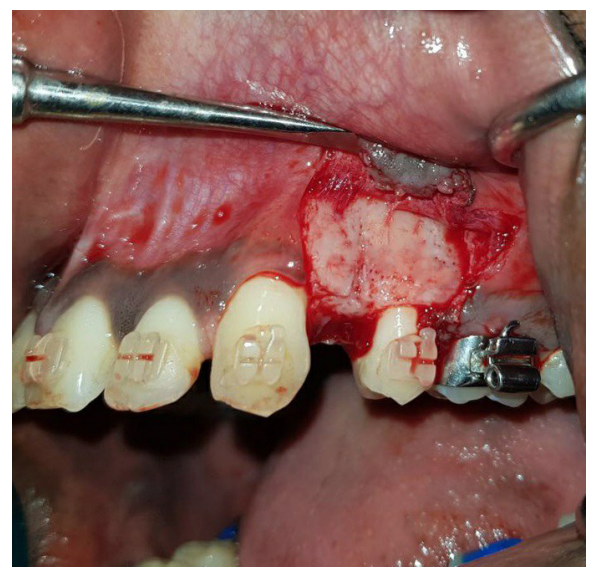

Fig.3: Incision and full thickness flap elevated.

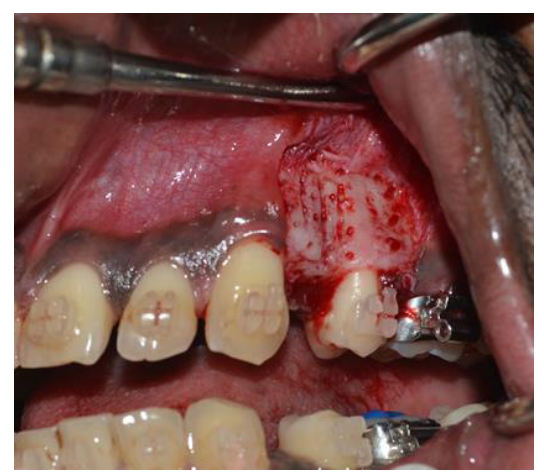

Fig.4: Vertical cuts and perforations done.

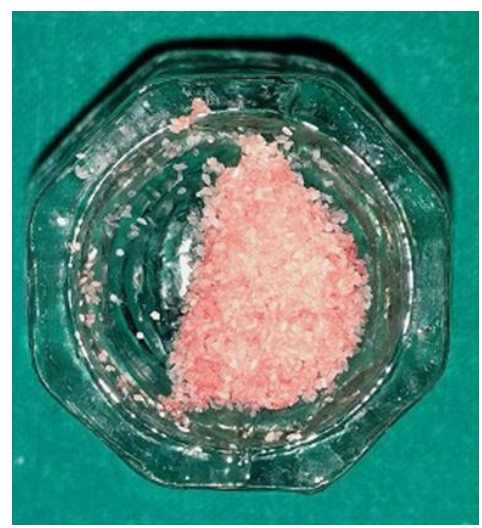

Fig.5: Bone graft mixed with $A-P R F$.

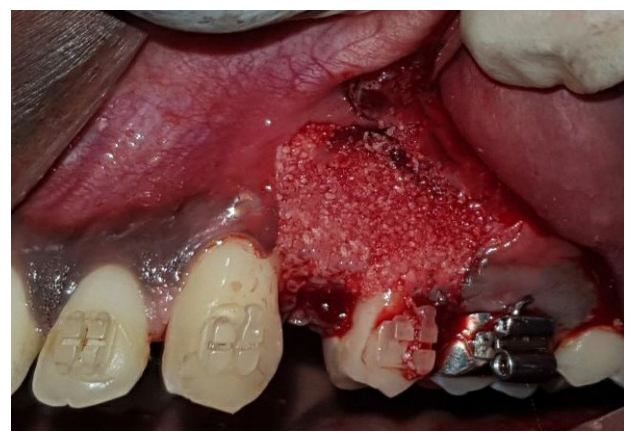

Fig.6: Mixture placed at the site.

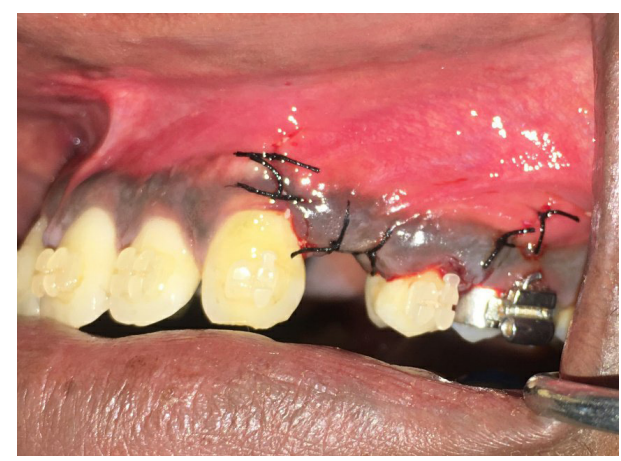

Fig.7: Sutures placed. 


\section{Discussion}

PAOO is considered important in the management of malocclusion to reduce the orthodontic treatment time by accelerating the space closure $[8,9]$. Corticotomy facilitated tooth movement was first described by Henrichkole, in which he explains the importance of preserving the intact spongiosa by this technique [10], while a total alveolar osteotomy may impair the intraosseous and intrapulpal blood circulation. After a healing period of 1 or 2 weeks orthodontic tooth movement is started. Demineralization occurs in the alveolar bone after corticotomy, and the remaining collagenous matrix of bone is transported with the tooth during its movement which will be remineralized following orthodontic movement [11]. Lee et al. and Sebaoun et al. reported systematic and histological evidence supporting the rapid tooth movement after alveolar corticotomy and explained that it was due to increase in anabolic and catabolic processes upto 21 days [12]. The fact that the teeth can be moved more rapidly, thus resulting in shortened treatment times, is certainly advantageous to the patient's periodontal health because less time in fixed appliances reduces patient "burnout" and substantially reduces the time available for relatively benign commensal bacterial biofilms to assume qualitative changes and convert to a destructive cytotoxic potential often seen when fixed appliances have remained on the teeth for more than 2 to 3 years.

The increased bone volume can provide more intact periodontium, a decreased need for extraction, a degree of facial reshaping, and an increase in the bony support for both the teeth and the overlying soft tissues $[12,13]$. The ability to increase the post-treatment bone volume and cover vital root surfaces can result in the repair of pre-existing alveolar dehiscences over root prominences and lessen the chance of new dehiscence formation, which can be a contributing factor to gingival recession.

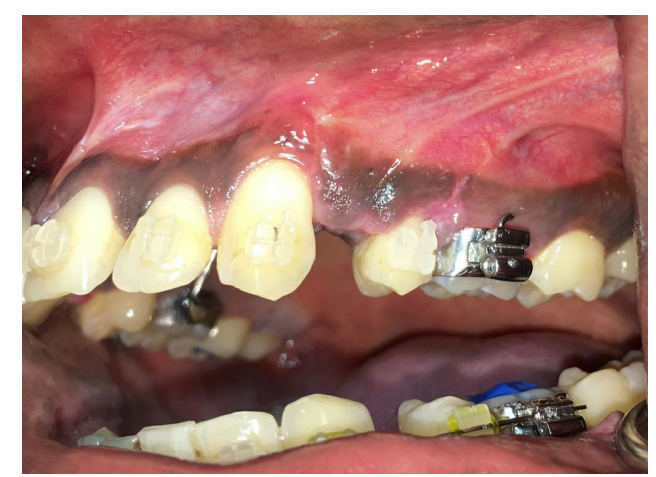

Fig.8: Post-operative view (10 days).

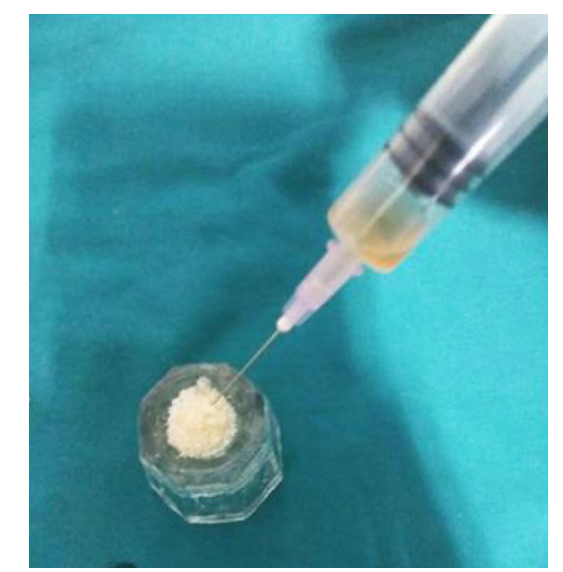

Fig.9: Bone graft mixing with $i-P R F$.

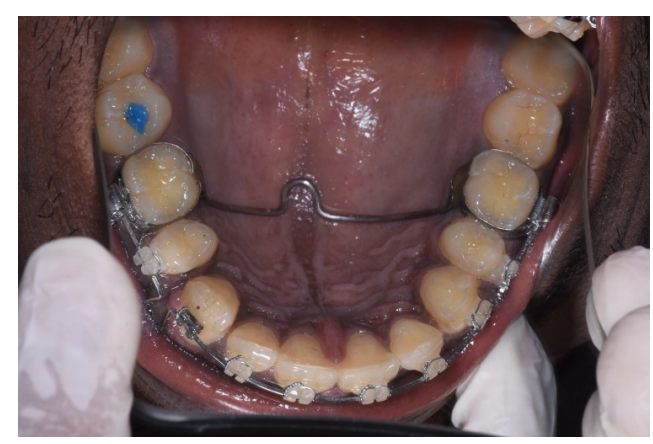

Fig.10: Post-operative view (3 months).

PRF is a proposed method for the use of platelet concentrate in which autologous growth factors derived from patients own blood further accelerate tissue regeneration. Major development and advancement were made with a liquid formulation of PRF which does not contain anticoagulant. Third generation super 
PRFs show superiority in effective and faster wound healing [14]. Ghanaati et al. introduced the low-speed concept for blood centrifugation whereby lower centrifugation speeds were shown to contain numerous cells, especially leukocyte [15]. Higher centrifugation force shift the cell population from the top to the bottom, thus by reducing centrifugation G-force, there is an increase in leukocyte number and they remain in the top layer. Decreasing the rpm while increasing the centrifugation time in the A-PRF group would have given an enhanced presence of neutrophilic granulocytes in the distal part of the clot. The granulocytes have tissue regeneration properties as well. They participate in the process of wound debridement by secreting several proteases, including matrix metalloproteinase 9 (MMP9), an extracellular matrix-digesting enzyme.

Furthermore, neutrophilic granulocytes expressing MMP9 play a part in the process of revascularization of the tissue defect by being recruited, eg. VEGF-A. Thus the distribution of neutrophilic granulocytes within the A-PRF clot might be the basis for a better functionality of the transplanted monocytes/macrophages and lymphocytes and their deployment to support tissue regeneration. i-PRF influence osteoblast behavior by inducing the migration, proliferation, and differentiation of human osteoblasts. One of the main benefits of i-PRF is the additional incorporation of leukocytes as well as fibrin proteins. i-PRF was capable of inducing higher cell migration and mRNA expression of TGF- $\beta$, PDGF, and COL1a. It was found that a $270 \%$ increase in fibroblast proliferation within 3 days and resulted in additional release of growth factors for rapid healing.

\section{Conclusion}

This case report presents with results of interdisciplinary amalgamation of PAOO technique with incorporation of A-PRF and i-PRF not only to address the rapid tooth movement but also to achieve rapid healing with adequate gingival architecture. Long-term studies are needed to evaluate the advantages and disadvantages of this technique as well as incorporation of minimally invasive additions and modifications to this technique.

Contributors: DR: manuscript writing, literature review and patient management; JK: critical inputs into the manuscript and patient management; JM, VR: literature review, discussion and imaging. JM will act as a study guarantor. All authors approved the final version of this manuscript and are responsible for all aspects of the study.

Funding: None; Competing interests: None stated.

\section{References}

1. Frost HM. The regional acceleratory phenomenon: a review. Henry Ford Hosp Med J. 1983;31:3-9.

2. Roubelakis MG, Trohatou O, Roubelakis A, Mili E, Kalaitzopoulous I, Papazolou G, et al. Platelet-rich plasma (PRP) promotes fetal mesenchymal stem/stromal cell migration and wound healing process. Stem Cell Rev. 2014;10:417-428.

3. Dohan DM, Choukroun J, Diss A, Dohan SL, Dohan AJ, Mouhyi J, et al. Platelet-rich fibrin (PRF): a secondgeneration platelet concentrate. Part I: technological concepts and evolution. Oral Surg Oral Med Oral Pathol Oral Radiol Endod. 2006;101:37-44.

4. Huang F, Yang S, Zhao J, Chang Y. Platelet-rich fibrin increases proliferation and differentiation of human dental pulp cells. J Endod. 2010;36:1628-1632.

5. Ghanaati S, Booms P, Orlowska A, Kubesch A, Lorenz J, Rutkowski J, et al. Advanced platelet-rich fibrin: A new concept for cell-based tissue engineering by means of inflammatory cells. J Oral Implantol. 2014;40:679-689.

6. Sinder BP, Pettit AR, McCauley LK. Macrophages: Their emerging roles in bone. J Bone Miner Res. 2015;30:2140-2149.

7. Jain V, Triveni MG, Kumar AB, Mehta DS. Role of platelet-rich- fibrin in enhancing palatal wound healing after free graft. Contemp Clin Dent. 2012;3:S240-S243.

8. M.S. Robles-Andrade C. Guerrero-Sierra C. HernándezHernández. Ortodonciaacelerada periodontal mente: fundamentosbiológicosytécnicasquirúrgicas. Rev Mex Periodontol. 2011;2:12-16.

9. Hassan AH, Al-Fraidi AA, Al-Saeed SH. Corticotomyassisted orthodontic treatment: review. Open Dent J. 2010;4:159-164.

10. Kole H. Surgical operations of the alveolar ridge to correct occlusal abnormlities. Oral Surg Oral Med Oral Pathol. 1989;12:515-520. 
11. Schilling T, Müller M, Minne HW, Ziegler R. Influence of inflammation-mediated osteopenia on the regional acceleratory phenomenon and the systemic acceleratory phenomenon during healing of a bone defect in the rat. Calcif Tissue Int. 1998;63:160-166.

12. Sebaoun JD, Kantarci A, Turner JW, Carvalho RS, Van Dyke TE, Ferguson DJ. Modeling of trabecular bone and lamina dura following selective alveolar decortication in rats. J Periodontol. 2008;79:1679-1688.

13. Wang L, Lee W, Lei DL, Liu YP, Yamashita DD, Yen SL.
Tisssue responses in corticotomy and osteotomy-assisted tooth movements in rats: Histology and immunostaining. Am J Orthod Dentofacial Orthop. 2009;136:770-776.

14. Priyanka N. Potdar, et al. SUPER PRFs- from L to i .Int J Res Dent. 2016;6:1-12.

15. Ghanaati S, Booms P, Orlowska A, Kubesch A, Lorenz J, et al. Advanced platelet-rich fibrin: a new concept for cell-base tissue engineering by means of inflammatory cells. J Oral Implantol. 2014;40:679-689. 